\title{
Die Adaptation der Entero-amylase an den chemischen Reiz. \\ Von
}

L. J. te Groen, med. cand. Assistent.

(Aus dem physiologischen Institut der Universität Amsterdam:

Direktor Prof. Dr. G. van Rynberk).

(Der Redaktion zugegangen am 5. Dezember 1913.)

1. Über die Anwesenheit einer Amylase im Darmsaft.

Seit Frerichs $\left({ }^{1}, 1846\right)$, der zum erstenmal die amylolytische Wirkung des Darmsaftes bei Hunden und Katzen festgestellt hat, sind viele einander widersprechende Untersuchungen über diesen Gegenstand ausgeführt worden. Röhmann $\left({ }^{2}, 1887\right)$ schließt nach einem eingehenden Literaturstudium, und nach eigenen Versuchen, daß Amylum im Dünndarm schnell umgesetzt und aufgesaugt wird. Die Erklärung der so weit auseinander laufenden Ansichten der Autoren, sucht er in der Tatsache, daß die verschiedenen Untersucher mit verschiedenen Abschnitten des Darmes arbeiteten. Auch ein neuer, ausgezeichneter Erforscher der Verdauungsfunktionen: U. Lombroso $(3,1912)$ bestätigt diese Hypothese.

Als Nebenursache möge noch die früher von Schiff $\left(^{4}\right.$, 1868) gemachte Annahme erwähnt werden, daß fehlerhaft angelegte Fisteln kein stärkespaltendes Enzym liefern sollen. Dazu kommt noch, daß viele Autoren den von ihnen benutzten Darmsaft durch chemische oder mechanische Reize erzielt hatten. Der so sezernierte Darmsaft ist wasserreich, jedoch fermentarm.

Aus der Literatur ersehen wir also, daß der Darmsaft eine Amylase enthält, wenn auch nicht alle Darmabschnitte in gleichem Maße an der Sekretion derselben beteiligt sind. Weiter ist festgestellt, daß der angewandte Reiz nicht gleichgültig ist. Hiermit kommen wir auf den eigentlichen Gegenstand meiner heutigen Untersuchung.

2. Über' die vermeintliche Adaptation von Enzymwirkungen im Darmkanal.

Das erste Béispiel einer vermeintlichen Adaptation von Enzymwirkungen im Darmkanal ist die bekannte Adaptation 
der Darmschleimhaut in bezug auf den Enterokinasegehalt. Über die Adaptation von anderen Fermentwirkungen im Darm ist wenig bekannt. Pawlow hat keine weiteren Untersuchungen darüber angestellt. Lombroso leugnet, daß es solche gäbe, auf Grund seiner Versuche an Vellaschen Fisteln. Er fand nämlich den Gehalt an Enzymen nicht verändert, wenn er seine Hunde mit verschiedenartiger Nahrung fütterte. Diese Versuchsanordnung scheint mir aber keine glückliche zu sein, wenigstens wenn es sich darum handelc, näheres über Adaptation des Darmes an direkte Reize zu ermitteln. Hat doch Lombroso in Übereinstimmung mit Shepovalnikow $(7,1911)$ gezeigt, was von Deelman $\left({ }^{8}, 1913\right)$ bestätigt worden ist, daß der Darm nur auf lokale Reize reagiert, und daß die Sekretion, die auf einen solchen Reiz auftritt, sich nicht auf entfernte Abschnitte ausdehnt.

Nur A mbard und Binet $\left({ }^{6}, 1908\right)$ schließen aus einigen sehr summarisch beschriebenen und daher kaum verwertbaren Versuchen, daß es keine Adaptation der Amylasesekretion im Darme gäbe. Dieser Frage habe ich versucht etwas näher zu treten.

\section{Eigene Versuche.}

Auf Anregung von Dr. Jansen, dem Abteilungschef für Biochemie des hiesigen physiologischen Laboratoriums, habe ich die Frage der eventuellen Adaptation der Amylasesekretion an Vellaschen Fisteln bei Hunden wieder aufgenommen. Statt jedoch, wie Lombroso, die eventuellen Veränderungen der enzymatischen Eigenschaften des sezernierten Saftes bei verschiedener Nahrung zu eruieren, habe ich die in Beziehung kommende Nahrung: Stärkekleister unmittelbar in die Fistel eingeführt, damit der chemische Reiz, worauf Adaptation stattfinden sollte, unmittelbar an der Schleimhaut angreifen kann.

a) Konstanz des Amylasegehalts.

In erster Linie war es nun natürlich unbedingt notwendig, festzustellen, ob der Amylasegehalt des Saftes meiner Fisteln konstant war. 
Ich hatte zu meiner Verfügung drei Fistelhunde. Hund I hatte eine einfache Vellasche Fistel, die schon mehr als ein Jahr vor Anstellung meiner Versuche angelegt ward. Hund II hatte eine Vellasche Doppelfistel, oder Omegafistel nach Lom broso, operiert am 15. Februar 1913. Hund III hatte eine einfache Vellasche Fistel angelegt am 24. Mai 1913.

Meine Versuche, die sich über mehrere Monate ausdehnten, wurden in der Weise angestellt, da $B$ Darmsaft aus den Fisteln aufgefangen wurde, 3 oder 4 mal pro die, also vor, während und nach der Mahlzeit. Die Hunde wurden in den nachfolgenden Perioden mit verschiedener Nahrung gefüttert. Jedesmal wurde die Fistel zuvor mit physiologischer Kochsalzlösung von etwa $37^{\circ}$ durchgespült, bis die herauskommende Flüssigkeit klar aussah. Dann wurde die Sekretion mittels $5 \mathrm{ccm}$ einer isotonischen Lösung von cholalsaurem Natrium angeregt. ${ }^{1}$ )

Von dem so erhaltenen Darmsaft wurden in zwei Röhrchen je $2 \mathrm{ccm}$ vermischt mit $5 \mathrm{ccm}$ einer 2\% igen Amylumlösung und 3 Tropfen Thymollösung. Die Röhrchen wurden nun eine Stunde in einem auf $38^{\circ} \mathrm{C}$. geheizten Thermostaten fortdauernd geschüttelt. Dann wurden einige Tropfen konzentrierte Kalilauge hinzugefügt, wonach die Fermentwirkung sofort einsetzen mußte.

Die in den Röhrchen entstandenen reduzierenden Körper bestimmte ich nach der Methode von Schoorl (11, 1899); obwohl diese Methode schon längst veröffentlicht worden ist, ist sie doch noch nicht allgemein bekannt, wenigstens in den Kreisen der Physiologen: in Abderhaldens sonst so ausgezeichnetem Handbuch der biochemischen Arbeitsmethoden wird sie nicht erwähnt. Es scheint mir daher zweckmäßig, das Prinzip dieser Methode hier in aller Kürze noch einmal anzugeben.

1) Von Lombroso $\left({ }^{10}, 1903\right)$ wurde festgestellt, daß ein Gemisch von Ölsäure und Galle auf den Dünndarm stark safttreibend wirkt. Dieser Saft ist seh' ' enzymreich (geprüft besonders auf Lipase). Dr. Jansen hat nun weiter im hiesigen Institut nachgewiesen $\left({ }^{\circ}, 1910\right)$, daß diese Wirkung auf den Gallensäuren beruht: man kann also das Lombrososche Gemisch mit ganz gleichem Resultat durch eine reine Lösung von einem cholalsauren Salz ersetzen. 
Die Schoorlsche Methode ist sehr genau und bequem, sogar leichter ausführbar als die von Bertrand. Das nach Kochen der Fehlingschen Lösung nicht niedergeschlagene Kupfer wird durch Hinzulügen von JK und nachher von verdünnter Schwefelsäure in $\mathrm{CuJ}$ und $\mathrm{J}$ umgesetzt. Das freiegewordene Jod wird sogleich mit 0,1-normaler Natriumthiosulfatlösung titriert. Mittels einer empirisch von Schoorl festgestellten Tabelle liest man dann ohne weiteres den Zuckergehalt ab.

Die Ergebnisse einer großen Reihe von Bestimmungen, die in oben angegebener Weise ausgeführt wurden, zeigten nun unzweideutig, daß der Amylasegehalt des Darmsaftes für jede Fistel konstant war. Im Laufe einer längeren Versuchsreihe nahm der Gehalt im ganzen jedoch ab, ebenso nahm er am selben Tage ab, wenn der Sekretionsreiz mehrere Male nacheinander wiederholt wurde. Ganz und gar wurden die Annahmen von Röhmann und Lombroso bestätigt, daß der Darmabschnitt, der den Saft liefert, von großer Bedeutung ist für die Enzymtätigkeit: je näher dem Duodenum, desto stärker die amylolytische Wirkung. Dies konnte ich sogar bei der Lombrososchen Omegafistel unzweideutig nachweisen: der aus der oralen Hälfte der Fistel, die ich mit A angeben werde, fließende Saft war immer stärker amylolytisch als der Saft, der aus der aboralen Hälfte (B) der gleichen Fistel aufgefangen wurde. Ich gebe hier einige Tabellen worauf diese Ergebnisse sich stützen:

\section{Tabelle I.}

Hund II. Viermalige Reizung pro die. Mittelwerte der in den zwei Röhrchen gefundenen Zahlen.

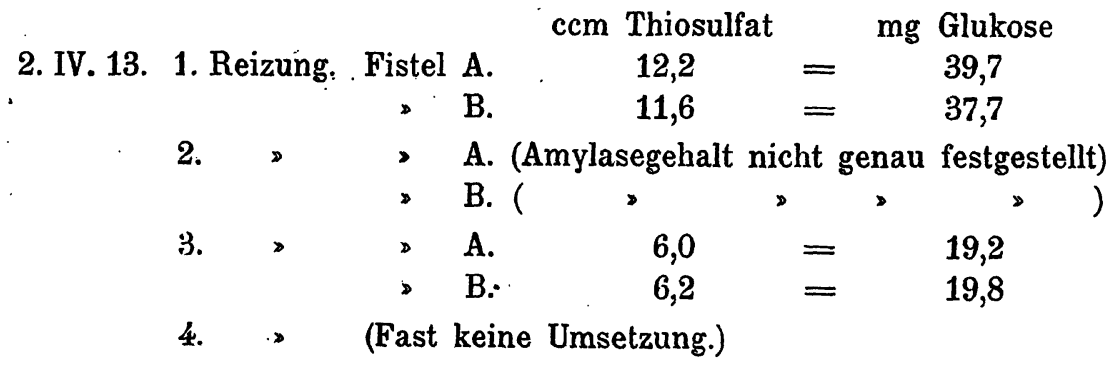


Die Adaptation der Entero-amylase an den chemischen Reiz. 95

\section{Tabelle II.}

Hund II. Zweimalige Reizung pro die.

31. III. 13. 1. Reizung. Fistel A.

$$
\text { ccm Thiosulfat mg Glukose }
$$
B.
$15,4 \quad=\quad 50,7$

(nicht ausgeführt)

2. > A. (Nicht ausgeführt)

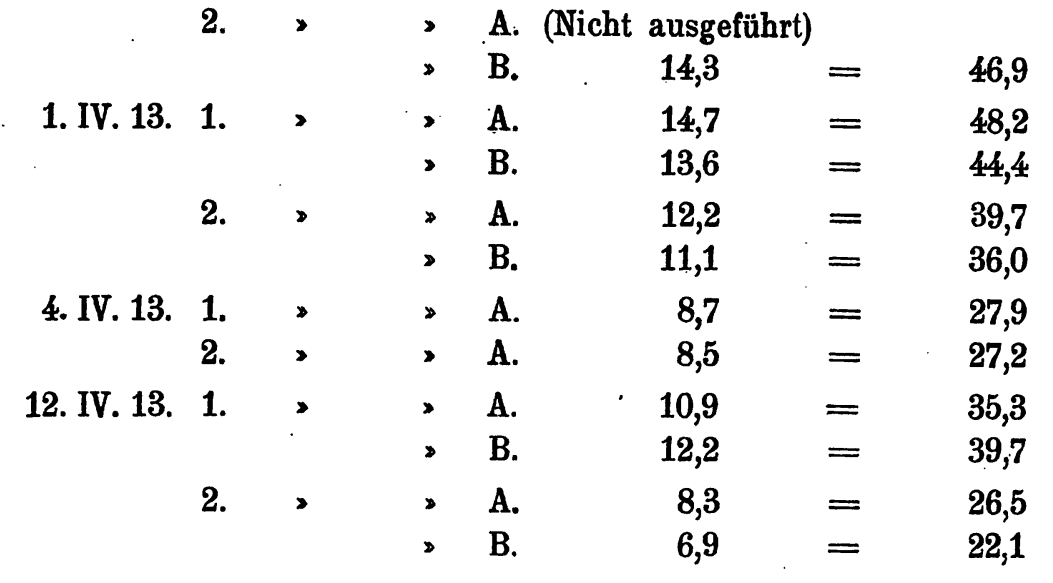

Tabelle III.

Hund II. Einmalige Reizung pro die.

3. IV. 13. Fistel A.

$\begin{array}{ccc}\text { ccm Thiosulfat } & & \text { mg Glukose } \\ 11,5 & = & 37,4 \\ 10,0 & = & 32,3 \\ 12,3 & = & 40,0 \\ 11,2 & = & 36,4 \\ 12,9 & = & 42,1 \\ 14,2 & = & 46,5 \\ 11,6 & = & 37,7 \\ 10,3 & = & 33,3 \\ 9,9 & = & 31,6 \\ 9,4 & = & 30,3\end{array}$

Es ergibt sich aus Tabelle I und II ohne weiteres, daß nach jeder Reizung die enzymatische Tätigkeit des gesammelten Saftes abnimmt. Diese Tabellen sind daher nicht ohne weiteres zur Beantwortung der Frage, ob die Enzymwirkung konstant ist; ' zu benutzen. Aus der Tabelle III geht dagegen die Konstanz der Enzymtätigkeit gut hervor. Nur sieht man hier deutlich, wie die orale A-Hälfte der Doppelfistel immer einen stärkeren Saft liefert als die aborale B-Hälfte. 
Auch wenn man die Zahlen, die während zwei Wochen erhalten wurden, wie sie täglich gefunden wurden für die gleiche Fistelhälfte vergleicht, sieht man deutlich, daß die Enzymwirkung abnimmt. Ich gebe hier die Zahlen von der ersten Hälfte des Monats April an.

Tabelle IV.

$\begin{array}{rcc}\text { Datum } & \begin{array}{r}\text { Hund II. Fistel A. } \\ \text { ccm Thiosulfat }\end{array} & \text { mg Glukose } \\ \text { 1. IV. } 1913 & 14.7 & 48,2 \\ \text { 2. }>1913 & 12,2 & 39,7 \\ \text { 3. }>1913 & 11,5 & 37,4 \\ \text { 4. }>1913 & 8,7(!) & 27,9(!) \\ 5 .>1913 & 10,7 & 34,7 \\ \text { 7. }>1913 & 12,3 & 40,0 \\ 10 .>1913 & 12,9 & 42,1 \\ 11 .>1913 & 11,6 & 37,7 \\ 12 .>1913 & 10,9 & 35,3 \\ 14 .>1913 & 9,8 & 31,6\end{array}$

Wie man sieht, sind die Zahlen ziemlich ähnlich. Natürlich gibt es Abweichungen: Pawlow $\left({ }^{5}, 1910\right)$ hat den Verdauungsapparat irgendwo mit einem chemischen Laboratotium verglichen. Wenn man nur alle Verhältnisse kennen würde, würde Pawlows Satz auch für den Amylasegehalt wohl gelten. Zurzeit gibt es noch unbekannte Faktoren, wodurch die Versuchsergebnisse von Zeit zu Zeit Sprünge zeigen, deren Erklärung vorläufig fehlt.

b) Adaptation der Amylasesekretion.

Nachdem ich also die relative Konstanz des Amylasegehalts des Darmsaftes der Fisteln festgestellt hatte, bin ich zur Prüfung der Frage, ob Adaptation bei dieser Sekretion einen Einfluß habe, übergegangen. Ich habe dazu Amylumlösung in die Fisteln eingeführt, um zu eruieren, ob in diesem Falle der sezernierte Saft mehr amylolytische Wirkung hatte als sonst.

Es hat sich nun herausgestellt, daß weder die Einführung einer Lösung von Amylum in Wasser ohne weiteres, noch einer solchen in physiologischer Kochsalzlösung den erwarteten 
Die Adaptation der Entero-amylase an den chemischen Reiz.

Erfolg hat, da die auf diese Flüssigkeiten sezernierten Saftmengen sehr klein sind. Ich habe daher ein isotonisches Gemisch von cholalsaurem Natrium und Stärkelösung benutzt. Mit dieser Lösung . habe ich an Hund II im Monat Mai 13 Versuche angestellt.

\section{Tabelle V.}

Hund II. Probereizung ohne Amylum.

9. V. 1913. Fistel A. $15,6 \mathrm{ccm}$ Thiosulfat $=51,4 \mathrm{mg}$ Glukose

$$
\text { B. } 14,2 \text {, > = } 46,5 \text {; , }
$$

Hund II. Reizung mit Na-Cholat und Amylumlösung.

13. V. 1913. Fistel A. $24,7 \mathrm{~cm}$ Thiosulfat $=85,4 \mathrm{mg}$ Glukose

$$
\text { B. } 23,8 \text {, },=81,8 \text {, }
$$

Hund II. Cholalsaures Natrium und Amylumlösung.

14. V. 1913. Fistel A. $21,7 \mathrm{ccm}$ Thiosulfat $=73,4 \mathrm{mg}$ Glukose.

Aus diesen Ergebnissen kann man ungezwungen auf eine Adaptation schließen. Bemerkenswert ist, daß der Gehalt an Amylase am 9. Mai, nachdem die Fistel während ungefähr drei Wochen nicht gereizt wurde, sich viel höher zeigte als normal. Trotzdem ist der Amylasegehalt am 13. Mai, nach Amylumreiz viel größer als jemals nach einfacher Reizung mit cholalsaurem Natrium von mir beobachtet wurde. Am folgenden Tag wurde wieder mit Amylum gereizt: dann war zwar der Amylasegehalt kleiner als das erste Mal, blieb immerhin stärker als nach einfacher Reizung mit cholalsaurem Natrium. Es scheint mir berechtigt, aus diesen Beobachtungen zu schließen, daß nach Einführung von Amylumlösung der Darm einen mehr Amylase enthaltenden Saft sezerniert, als das sonst auf eine stark sekretionsanregende Flüssigkeit hin der Fall ist. Das Amylum wirkt also sozusagen als ein spezifischer Reiz für die Amylase-Sekretion.

c) Weitere Versuche.

Obenerwähnte Versuche wurden alle mit dem Hunde II (Lombrososche Omegafistel) angestellt. Zur Kontrolle habe ich gleichartige Untersuchungen auch bei zwei anderen Hunden ausgeführt. Dies umsomehr, weil der Hund II eine ansteckende Hautkrankheit bekam und getötet wurde.

Ich bringe die Ergebnisse in einigen Tabellen:

Hoppe-Sepler's Zeitschrift f, physiol. Chemie. LXXXIX. 


\section{Tabelle VI.}

Hund I. Einfache Reizung mit isotonischer Na-Cholatlösung, zor Bestimmung der Konstanz des Amylasegehalts.

6. VI. $19136,6 \mathrm{ccm}$ Thiosulfat $=21,1 \mathrm{mg}$ Glukose

7. > 19138,3 > , $=26,6$, ,

9. 19136,6 , > $=21,1$, ,

19. 19135,4 , , $=17,2$, ,

\section{Tabelle VII.}

Hund III. Einfache Reizung mit isotonischer Na-Cholatlösung; zur. Bestimmung des normalen Amylasegehaltes.

\begin{tabular}{|c|c|c|c|c|c|c|c|}
\hline 9. & VI. 1913 & 8,7 & $\mathrm{ccm}$ & Thiosulfat & $=27,9$ & & Glukose \\
\hline 10. & > 1913 & 5,7 & > & , & $=18, \tilde{5}$ & $>$ & 2 \\
\hline 11. & , 1913 & 6,6 & , & , & $=21,1$ & > & , \\
\hline 16. & > 1913 & 8,4 & > & $>$ & $=26,9$ & $>$ & , \\
\hline 19, & \ 1913 & 7,7 & $\gg$ & $\gg$ & $=24,7$ & ? & , \\
\hline
\end{tabular}

Auch bei diesen beiden Hunden zeigte sich der Amylasegehalt des Fistelsaftes alșo ziemlich gleich. Nachdem ich dies festgestellt hatte, schritt ich zum zweiten Teile des Problems: ob der Gehalt an Amylase sich bedeutend ändere nach spezifischer Reizung mit Amylum.

$\mathrm{Zu}$ diesem $\mathrm{Zwecke}$ versuchte ich erst eine isotonische Mischung von cholalsaurem Natrium und Stärkelösung zu verwenden. Da jedoch beide Hunde dabei keine genügenden Quantitäten Saft sezernierten, kam ich dazu, meine Versuchstechnik in folgender Weise zu ändern: Die Fistel wurde mit physiologischer Kochsalzlösung von etwa $37^{\circ} \mathrm{C}$. durchgespült und dann mit $5-10 \mathrm{ccm}$ konzentrierter Stärkekleister, gelöst in physiologischer Kochsalzlösung, injiziert. Nach 20-40 Minuten (während welcher Zeit die Hunde frei herumliefen) reizte ich den Darm mit $5 \mathrm{ccm}$ isotonischer Lösung von cholalsaurem Natrium. Der dann aufgefangene Darmsaft wurde auf die schon erwähnte Weise auf Amylase geprüft. Da man jedoch bei diesem Verfahren fürchten mußte, daß etwa Stärkekleister oder sogar Glukose von der ersten Injektion bei der zweiten Sekretion mit heraus befördert werden könnte, nahm ich immer eine Probe des ausfließenden Saftes und prüfte sie sowohl auf Amylum (Jodprobe), als auf Glukose (Fehlingsche Probe). Diese Kontrollproben fielen immer negativ aus. Dies bedeutet, 
Die Adaptation der Entero-amylase an den chemischen Reiz. 99

daß vom erst injizierten Amylum nach 20-40 Minuten kaum mehr im Darm etwas übrig war, sondern dåß alles abgebaut und als Glukose aufgesaugt war.

\section{Tabelle VIII.}

Hund I. Versuche auf die Adaptation für Amylase.

14. VI. $191316,1 \mathrm{ccm}$ Thiosulfat $=53,2 \mathrm{mg}$ Glukose

20. , 191312,4 , > $=40,4$, >

23. 191319,5 , > $=65,1$, ।

24. > 191314,8 , > $=48,6$, ,

25. > 191315,6 > $>51,1$, ।

26. $>191310,6>\quad>=34,3$ > ।

27. 191316,9 , $1=55,9$, ,

Tabelle IX.

Hund III. Versuche zur Eruierung einer eventuellen Adaptation der Amylase-Sekretion seitens des Dünndarms.

14. VI. $191318,2 \mathrm{~cm}$ Thiosulfat $=60,5 \mathrm{mg}$ Glukose

17. 191313,2 , > = 43,1 , ,

18. , 191313,8 , , $=45,1$, ,

20. > 191313,6 > > $=44,4$ > ।

24. > 191312,2 > > $=39,7$ ». ॥

25. > 191310,3 , (!) > = 33,3 >(!) ,

26. $19139,->(!), \quad=28,9>(!)$,

27. . 191315,7 , > $=49,7$, "

Man ersieht aus diesen Tabellen eine sehr deutliche Vermehrung des Amylasegehaltes. Dies ist um so merkwürdiger, als der spezifische Reiz 20-40 Minuten zuvor stattgefunden hatte. Diese Versuchsergebnisse sagen also nicht nur aus, daß auf spezifische Reizung mit Amylum ein starker amylolytischer Saft sezerniert wird, sondern daB die Adaptation einige Zeit nach stattgefundener Reizung fortdauert.

Zusammenfassung und Schlußfolgerungen.

1. Darmsaft, aus Vellaschen Fisteln des Dünndarms erhalten, hat amylolytische Wirkung, auch wenn er nach nichtspezifischer Reizung (cholalsaurem Natrium) sezerniert worden ist.

2. Bei Vergleich des Amylasegehaltes von Darmsaft, aufgefangen aus einer Lombrososchen Omega-Doppelfistel, ergab 
sich konstant, daß der aus der oralen Hälfte der Fistel fließende Saft amylasereicher war, als der aus der aboralen Hälfte aufgefangene.

3. Der Amylasegehalt ist für jede Fistel in verschiedenen Nahrungsperioden mit verschiedenem Futter annähernd konstant.

Bei mehrmaliger Reizung, sei es am selben Tage, sei es auch in einer längeren Zeitperiode täglich wiederholter Versuche, nimmt der sonst konstante Amylasegehalt stetig ab.

4. Unmittelbare Reizung der Darmschleimhaut mit Stärkelösung ruft eine Sekretion hervor, die unzweideutig amylasereicher ist als in der Norm. Die Vermehrung des Amylasegehaltes hält nach stattgefundener Reizung mit Amylum einige Zeit an.

Nach dem Vorgehenden wäre also eine Adaptation der Entero-Amylase sichergestellt. Man sollte geneigt sein, dem Darmsaft in dieser Beziehung eine bedeutendere Funktion zuzuschreiben, als "Pancreas-adjuvans», wie $\mathrm{Pa}$ w low es annimmt.

\section{Literatur.}

1. F. Th. Frerichs, Die Verdauung, Wagners Handwörterb. der Thys., Braunschweig 1896, Bd. 3, S. 658.

2. F. Röhmann, Über Sekretion und Resorption im Dünndarm. Pflügers Arch., Bd. 41, S. 424 (1887).

3. U. Lombroso, Les activités enzymatiques de la sécrétion entérique, Archives Italiennes de Biol., Bd. 57, S. 317 (1912).

4. M. Schiff, Nuove ricerche sul potere digerente del succo enterico. Il Morgagni, 1867. Ref. Centralbl. f. d. Med. Wissensch., 1868, S. 357.

5. J. P. Pawlow, The work of the digestive Glands, London 1910, S. 2.

6. L. Ambard et M. E. Binet, Quantités d'amylase contenues' dans le tube digestif aux différents moments de la digestion et au cours d'alimentations diverses, C. R. de la Soc. biol., Vol. 64, H. 6 (1908).

7. U. Lombroso, La sécrétion entérique, Arch. Ital. de Biol., Bd. 56, S. 17 (1911).

8. H. T. Deelman, Eenige proeven met omega-Darmfistels volgens Lombroso, by honden, Nederl. Tydschr. v. Geneesk., 1913, Bd. 2, S. 687.

9. B. C. P. Jansen, Beitrag zur Kenntnis der Enterolipase, Diese Zeitschrift, Bd. 68, S. 400 (1910).

10. U. Lombroso, Atti del Congresso di Patologia in Firenze 1903.

11. N. Schoorl, Zeitschr. f. angew. Chemie, 1899, S. 635. 